\title{
Nonthermal processes in microquasars
}

\author{
Valentí Bosch-Ramon \\ Dublin Institute for Advanced Studies, 31 Fitzwilliam Place, Dublin 2, Ireland \\ email: valenti@cp.dias.ie
}

\begin{abstract}
Microquasars are X-ray binaries that show extended radio jets. These jets can accelerate particles up to relativistic energies that produce non-thermal emission from radio to $\mathrm{TeV}$, and could also make a non-negligible contribution to the galactic CRs in some energy ranges. The orbital motion and compactness of these sources allow the study of high-energy astrophysical phenomena in extreme conditions that change in accessible timescales. In this work, I briefly discuss the production of broadband non-thermal emission in microquasars, putting special emphasis on the high- and the very high-energy bands.
\end{abstract}

Keywords. Microquasars, non-thermal processes, gamma-rays

\section{Introduction}

Microquasars are X-ray binaries with non-thermal radio jets (see, e.g. Mirabel \& Rodríguez 1999, Ribó 2005). They are called high-mass microquasars (HMMQ) when hosting a massive star, and low-mass microquasars (LMMQ) otherwise. The supply of energy in microquasars can be either accretion or an accreting rotating black-hole, and the power is channelled to a jet that is launched from the inner regions of the accretion disk (e.g. Blandford \& Znajek 1977, Blandford \& Payne 1982).

Historically, microquasars have been considered strong candidates to gamma-ray sources (e.g. Chadwick et al. 1985; see also Chardin \& Gerbier 1989, Levinson \& Blandford 1996, Paredes et al. 2000). However, it has not been until the most recent generation of ground-based Cherenkov telescopes arrived, like HESS, MAGIC and VERITAS, and satellite-borne instrumens, like Fermi and AGILE, that microquasars have become fully recognized as powerful gamma-ray emitters. The most relevant cases are the microquasars Cygnus X-1 and Cygnus X-3 (Albert et al. 2007, Sabatini et al. 2010, Tavani et al. 2009a, Abdo et al. 2009c; for upper-limits in some energies or source states, see Del Monte et al. (2010), Saito et al. 2009, Aleksic et al. 2010). Although the detected radio emission is evidence of particle acceleration in microquasar jets, the finding of microquasars being gamma-ray emitters is the proof that these sources can very efficient channelling accretion or black-hole rotational energy into radiation. In this paper, we review different aspects related to non-thermal emission in microquasars, focusing mainly in the production of gamma-rays.

\section{Non-thermal emission in microquasars}

Microquasar jets can produce non-thermal populations of relativistic particles via diffusive shock acceleration or other mechanisms at different spatial scales (for a recent review, see Bosch-Ramon 2010a; see also, e.g., Romero et al. 2003, Paredes et al. 2006, Rieger et al. 2007), generating photons of energies from radio to gamma-rays through synchrotron and inverse Compton (IC). Gamma-rays can be also produced through hadronic interactions, like proton-proton $(p p)$ interactions, photomeson production, or photodisintegration. We refer the reader to the work by Bosch-Ramon \& Khangulyan (2009) 


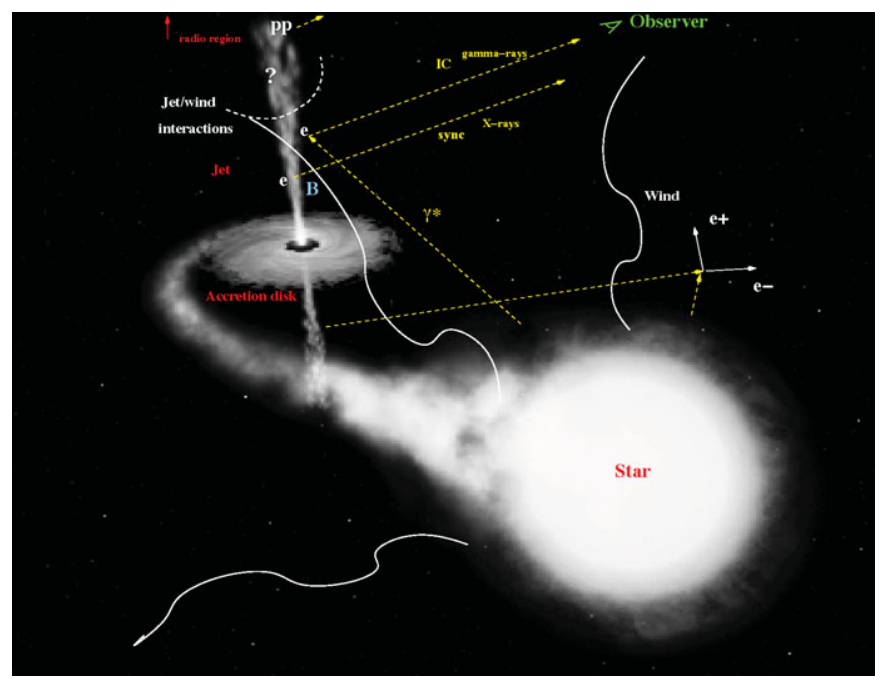

Figure 1. Illustrative picture of the microquasar scenario, in which relevant elements and processes are shown (background image from ESA, NASA, and Félix Mirabel).

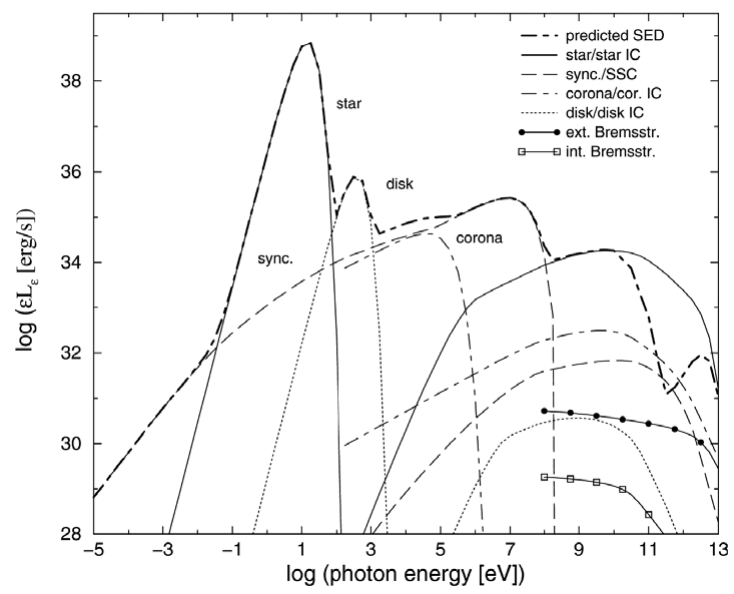

Figure 2. Computed spectral energy distribution for a typical HMMQ (see Bosch-Ramon et al. 2006a).

(and references therein) for details on non-thermal processes in the present context. In microquasars, the corona (or the base of the jet) could be also a non-thermal emitting region (e.g. Gierlinski et al. 1999; see also Romero et al. 2010), as well as the termination region of the jet in the ISM (e.g. Bordas et al. 2009). The relativistic protons that may be accelerated in microquasars can escape these systems enriching the CR content of our galaxy (e.g. Heinz \& Sunyaev 2002). A sketch of the microquasar scenario, with the main elements and processes relevant to microquasars, is shown in Fig. 1.

\subsection{High-mass microquasars}

In the context of HMMQs, likely the most efficient gamma-ray mechanism would be IC scattering of photons from the stellar companion (e.g. Paredes et al. 2000, Dermer \& Böttcher 2006, Bosch-Ramon et al. 2006a). As discussed in Sect. 3, the presence of the stellar photons may also imply strong attenuation of the gamma-ray emission. Hadronic 


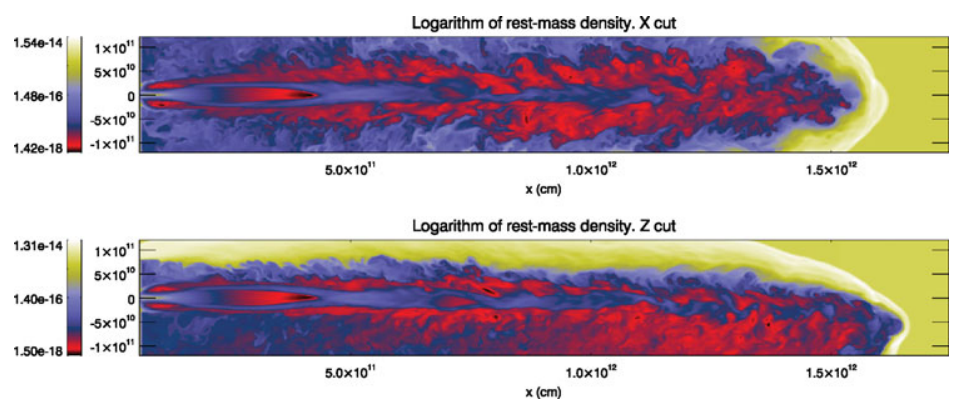

Figure 3. Two-dimensional map of the density of a jet interacting with the stellar wind, which is coming from the top of the image (see Perucho et al. 2010).

processes have been also discussed in the past (e.g. Romero et al. 2003; Aharonian et al. 2006b), although their efficiency is expected to be smaller than that of stellar photon IC. In Fig. 2 we show the computed broadband spectral energy distribution of HMMQs. In this particle instance, the dominant non-thermal emission processes are synchrotron and stellar photon IC.

Beside the jet itself, stellar wind-jet dynamical interactions at the binary spatial scales could also lead to non-thermal emission in HMMQs (Perucho \& Bosch-Ramon 2008), and the likely clumpy nature of the wind (e.g. Owocki \& Cohen 2006) could lead to HE and VHE flares (see, e.g., Araudo et al. 2009). An example of the importance of the wind-jet interaction is shown in Fig. 3, in which it is shown a 2-dimensional map of the density of a jet of power $10^{35} \mathrm{erg} \mathrm{s}^{-1}$ interacting with the stellar wind, which is coming from the top. Note that the jet is suffering significant bending and strong disruption not far beyond an asymmetric recollimation shock. Jets significantly more powerful, of up to $10^{37} \mathrm{erg} \mathrm{s}^{-1}$, may be still affected by jet disruption due to the growth of non-linear instabilities triggered in the recollimation shock (see Perucho et al. 2010).

\subsection{Low-mass microquasars}

Low-mass microquasars have been also proposed to be HE and VHE emitters. In these objects, the most efficient gamma-ray mechanisms could be external IC with accretion photons, synchrotron self-Compton or hadronic interactions in the inner-most regions of the jet base (e.g. Atoyan \& Aharonian 1999, Bosch-Ramon et al. 2006a, Romero \& Vila 2009). In Fig. 4, we show the computed spectral energy distribution for a powerful jet of a low-mass microquasar. The dominant IC component is either IC scattering off corona photons or synchrotron self-Compton; note the soft spectrum above GeV energies due to the Klein Nishina effect. Gamma-ray absorption in the accretion disk and corona fields can be significant in this context (for details, see Bosch-Ramon et al. 2006b).

\section{Impact of gamma-ray absorption}

In high-mass microquasars, if gamma-ray absorption takes place in the photon field of the star (e.g. Ford 1984, Protheroe \& Stanev 1987, Moskalenko \& Karacula 1994, Böttcher \& Dermer 2005, Dubus 2006a, Khangulyan et al. 2008, Reynoso et al. 2008), the whole binary system could be an efficient broadband non-thermal emitter. In such a case, the created pairs can radiate through synchrotron and IC emission in the whole spectral band, interacting with the ambient magnetic field and stellar photons, respectively (Bosch-Ramon et al. 2008a). If the energy density of the magnetic field were much 


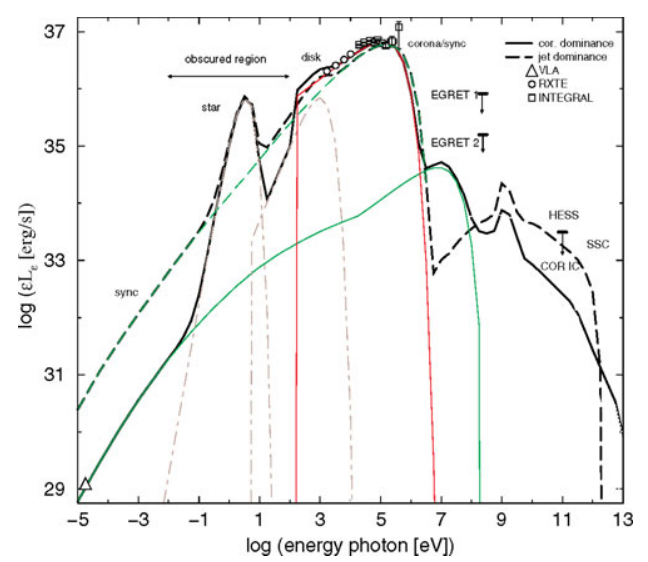

Figure 4. Computed spectral energy distribution of the non-thermal emission from $1 \mathrm{E}$ 1740.7-2942 for two situations. In one case, the hard X-rays come from a corona (long dashed line), whereas in the other, they are of synchrotron origin and come from the jet. Absorption in the accretion disk and corona photon fields has been taken into account (see the two dips below and above $\sim \mathrm{GeV}$ energies). For details, see Bosch-Ramon et al. (2006b).

smaller than that of the stellar photon field, IC scatterings would be the dominant cooling channel of these pairs. Then these pairs would produce more gamma-rays that to their turn may be also absorbed, triggering what is called an electromagnetic cascade. In this way, the effective optical depth to gamma-rays would be significantly reduced. The deflection of the created pairs in the ambient magnetic field determines whether the cascade is linear or three dimensional (e.g. Bednarek 1997, Aharonian et al. 2006b, Orellana et al. 2007, Sierpowska-Bartosik \& Torres 2007, Zdiziarski et al. 2009, Cerutti et al. 2010).

Under strong enough magnetic fields, electromagnetic cascades get suppressed (Khangulyan et al. 2008) and the X-ray (synchrotron) and GeV (single-scattering IC) emission from secondary pairs dominates the secondary energy output (Bosch-Ramon et al. 2008a). In Figs. 5 and 6 we show, as an example of this, the spectral energy distribution of the secondary radiation for a binary with system properties similar to those of LS 5039 (Aragona et al. 2009), computed for the phases when the compact object is in front (inferior conjunction) and behind the star (superior conjunction). The magnetic field in the stellar surface has been fixed to $20 \mathrm{G}$.

For stronger magnetic fields, the secondary radio emission may be also significant (Bosch-Ramon 2009, Bosch-Ramon 2010b). In Fig. 7, we show $5 \mathrm{GHz}$ radio images for a similar case but adopting a star magnetic field of $200 \mathrm{G}$. The images for four different orbital phases are presented: 0.0 (superior conjunction), 0.25, 0.5 (inferior conjunction), and 0.75 .

\section{Microquasars in context: jet sources and gamma-ray binaries}

The phenomenology at high energies of microquasars can be semi-quantitatively explained accounting for a set of ingredients or physical processes and effects: strong shocks; the angular dependence of gamma-ray absorption and IC scattering and the role of internal and external absorption (Bosch-Ramon et al. 2006a; Khangulyan et al. 2008), in some cases locating the emitter at some height above the orbital plane (for acceleration and modeling arguments, see Khangulyan et al. 2008; for absorption arguments, see Bosch-Ramon et al. 2008b); adiabatic losses (e.g. Bosch-Ramon et al. 2006c). The 


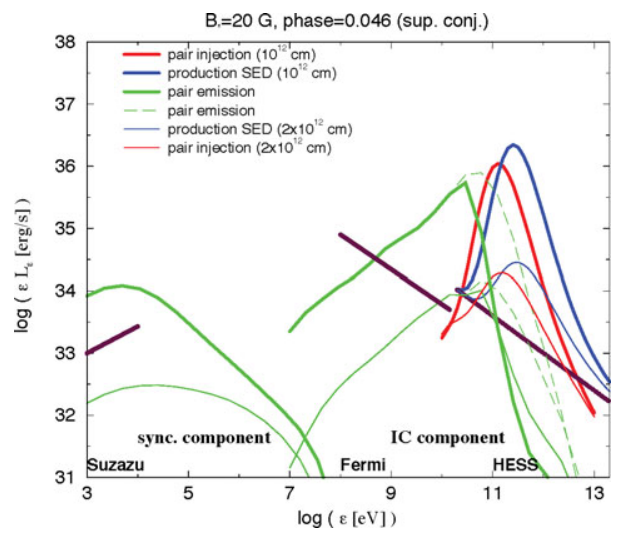

Figure 5. Computed spectral energy distribution of the radiation produced by secondary pairs in a high-mass binary (green lines) at the orbital phase associated to the superior conjunction of the compact object. Two different configurations have been considered, one in which the emitter is at a height of $10^{12} \mathrm{~cm}$ (production gamma-ray spectrum: thick solid blue line; corresponding pair injection: thick solid red line), and at $2 \times 10^{12} \mathrm{~cm}$ (production gamma-ray spectrum: thin solid blue line; corresponding pair injection: thin solid red line) above the compact object. The production spectral energy distribution (thin long-dashed) of the secondary IC emission is also shown.

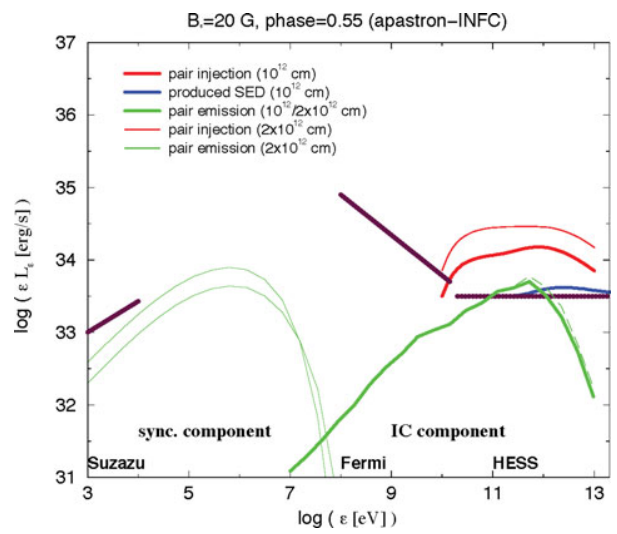

Figure 6. The same as in Fig. 5 but for the inferior conjunction of the compact object.

role of the magnetic field, e.g. through the impact of synchrotron cooling, is crucial for the VHE spectrum and luminosity. As noted above, one should not neglect either the impact of the stellar wind on the non-thermal processes (Perucho et al. 2010). Finally, the emitting region, generally the jet, can move fast enough to require the inclusion of Doppler boosting effects.

Beside microquasars, there are other jet sources in the Universe, like young stellar objects, active galactic nuclei and gamma-ray bursts. Together with microquasars, all these jet sources share the same basic elements: an accreting object; particle acceleration, possibly through strong shocks, Doppler boosting; and, with some exceptions, the same main suspects to be the dominant radiation processes (synchrotron, IC, and perhaps hadronic interactions). Nevertheless, despite being so similar, there are still important differences between the mentioned sources, like the accretor, the distance to us, the timescales, the accretion rate, and the properties of the jet itself, like its velocity and beaming relevance, density and temperature (e.g. efficient/unefficient thermal emission). There are as 

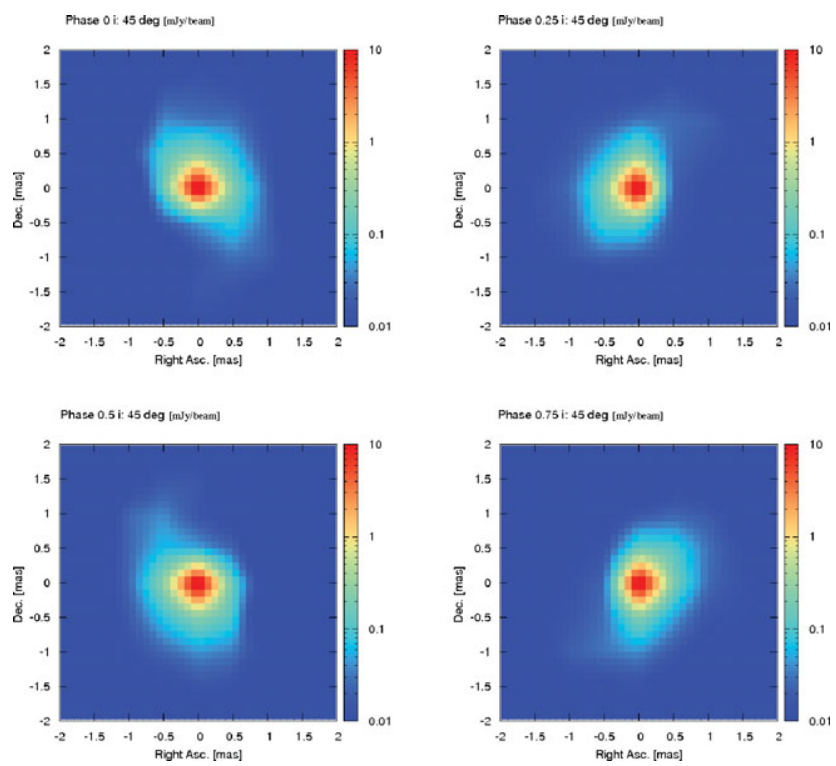

Figure 7. Computed image of the $5 \mathrm{GHz}$ radio emission, in the direction to the observer, for different orbital phases. Units are given in mJy per beam, being the beam size $\sim 1$ milliarcsecond.

well the environmental factors, like external sources of photons and the external medium density and velocity. For instance, in the case of AGNs, different powers and medium densities give rise to different jet termination structures, with instabilities having a very important role (e.g. Fanaroff \& Riley 1974; Perucho et al. 2010b). Also, GRBs can show very different phenomena during their afterglow phase, when the jet terminates, depending on the characteristics of the environment, since it may be determined by a massive star wind for a collapsar long GRB, or could be a much less dense medium in the case of coalescence of two compact objects (Chevalier \& Li 1999). Finally, in young stellar objects, the jet termination region may be the best candidate to be a gamma-ray emitter, given the strong shocks occuring there (e.g. Araudo et al. 2007, Bosch-Ramon et al. 2010). The magnetization level of the jet also determines the acceleration processes and the emitting mechanism, and may be different in each source or emitting region.

Microquasars are a subclass of gamma-ray emitters that pertain to the more general class of gamma-ray binaries. These latter sources are binary systems in which gamma-ray production takes place, and generally consist of a normal (non-degenerated) star and a compact object or another normal star. In addition to microquasars (Cygnus X-1 and Cygnus X-3), there is one confirmed high-mass binary hosting a non-accreting pulsar, PSR B1259-63 (Aharonian et al. 2005b), possibly a massive star binary, $\eta$-Carina (Tavani et al. 2009b), two high-mass binaries harboring a compact object of unknown nature, LS 5039 and LS I +61 303, (Kniffen et al. 1997, Paredes et al. 2000, Aharonian et al. 2005a, Aharonian et al. 2006a, Albert et al. 2006, Abdo et al. 2009a, Abdo et al. 2009b; for discussions on the nature of these sources, see, e.g., Dubus 2006b, Chernyakova et al. 2006, Romero et al. 2007, Bosch-Ramon \& Khangulyan 2009), and a high-mass binary candidate, HESS J0632+057 (Aharonian et al. 2007). Very recently, HE emission concurrent with a nova in the Symbiotic Binary V407 Cygni has been detected by Fermi Abdo et al. 2010, incorporating symbiotic binaries to the list of gamma-ray binary classes. All gamma-ray binaries share the main ingredients for gamma-ray production, i.e. powerful 
supersonic outflows, which may be collimated or quasi-spherical; strong shocks leading to particle acceleration; dense ambient photon fields for IC (although matter targets for hadronic processes may be important in some cases); and gamma-ray absorption in dense ambient photon fields.

As shown in the previous paragraph, physical effects and processes occurring in microquasars are also present to different extents in the known types of gamma-ray binaries and jet sources. In the context of gamma-ray binaries, these similarities make in some cases the identification of the specific nature of the object a difficult task, as it is shown by the debate on the nature of LS 5039 and LS I +61 303 (i.e. HMMQs versus nonaccreting pulsar high-mass binaries, if not a really new class of object). In case of jet sources, similarities can help to understand sources whose (e.g.) timescales are not accessible to us. However, the similarities should not mask the differences, which put limits to analogy arguments that sometimes can be misleading. In order to avoid to carry analogies too far, and to shed light on complicated cases of source nature confusion, it is of primary importance to apply comprehensive approaches to study theoretically these objects, including semi-analytical modeling, numerical calculations (secondary emission) and (magneto)hydrodynamical simulations, all this encompassed of course by simultaneous multiwavelength observations with high angular, spectral and time resolution.

\section{Acknowledgements}

The author acknowledges support of the Spanish MICINN under grant AYA200768034-C03-1 and FEDER funds. The author also acknowledges support of the European Community under a Marie Curie Intra-European fellowship.

\section{References}

Abdo, A. A., et al. 2009b, ApJ, 706, L56

Abdo, A. A., et al. 2009b, ApJ, 701, L123

Abdo, A. A., et al. 2009c, Science, 326, 1512

Abdo, A. A., et al. 2010, Science, 2010, Science, 329, 817

Acciari V. A., et al., 2008, ApJ, 679, 1427

Aharonian, F., et al. 2005a, Science, 309, 746

Aharonian, F., et al. 2005b, A\&A A, 442, 1

Aharonian et al. 2006a, J. Phys. Conf. Ser., 39, 408 [astro-ph/0508658]

Aharonian, F., et al. 2006b, A\&SA, 460, 743

Aharonian, F., et al. 2007, A\&SA, 469, L1

Albert, J., et al. 2006, Science, 312, 1771

Albert, J., et al. 2007, ApJ, 665, L51

Aleksic, J. 2010, ApJ, 721, 843

Aragona, C., et al. 2009, ApJ, 698, 514

Araudo, A. T., et al. 2007, A\& A, 476, 1289

Araudo, A. T., Bosch-Ramon, V., \& Romero, G. E. 2009, A\&A, 503, 673

Atoyan, A. M. \& Aharonian, F. A. 1999, MNRAS, 302, 253

Bednarek, W. 1997, A\& A, 322, 523

Blandford, R. D. \& Znajek, R. L. 1977, MNRAS, 179, 433

Blandford, R. D. \& Payne, D. G. 1982, MNRAS, 199, 883

Bordas et al. 2009, A\&\&A, 497, 325

Bosch-Ramon, V., Romero, G E., \& Paredes, J. M. 2006a, A\&A, 447, 263

Bosch-Ramon, V. et al. 2006b, A\&A A, 457, 1011

Bosch-Ramon, V., Paredes, J. M., Romero, G. E., \& Ribó, M. 2006, A\&\&A, 459, L25

Bosch-Ramon, V., Khangulyan D., \& Aharonian F. A. 2008a, A $\mathscr{J} A, 482,397$

Bosch-Ramon, V., Khangulyan D., \& Aharonian F. A. 2008b, A $\mho A$, 489, L21 
Bosch-Ramon, V. 2009, A\&A A, 493, 829

Bosch-Ramon, V., \& Khangulyan, D. 2009, Int. Jour, of Mod. Phys. D, 18, 347

Bosch-Ramon, V. 2010a, ASPC, 422, 13

Bosch-Ramon, V. 2010b, IJMPD, 19, 741

Bosch-Ramon, V. 2010c, $A \mathscr{G} A, 511,8$

Böttcher, M. \& Dermer, C. D. 2005, ApJ, 634, L81

Chadwick, P. M., et al. 1985, Nature, 318, 642

Chardin, G. \& Gerbier, G. 1989, A\& A, 210, 52

Chernyakova, M., Neronov, A., \& Walter, R. 2006, MNRAS, 372, 1585

Chevalier, R. A. \& Li, Z. 1999, ApJ, 520, 29

Dermer, C. \& Böttcher, M., ApJ 643, 1081 (2006)

Dubus, G. 2006a, $A \mathscr{E} A, 451,9$

Dubus, G. 2006b, $A \mathscr{E} A$, 456, 801

Fanaroff, B. L. \& Riley, J. M. 1974, MNRAS, 167, 31

Ford, L. H. 1984, MNRAS, 211, 559

Gierlinski et al. 1999, MNRAS, 309, 496

Heinz \& Sunyaev 2002, A\&A, 390, 751

Khangulyan, D. et al. 2007, MNRAS, 380, 320

Khangulyan, D., Aharonian, F., \& Bosch-Ramon, V. 2008, MNRAS, 383, 467

Kniffen, D. A. et al. 1997, ApJ, 486, 126

Levinson, A. \& Blandford, R. D. 1996, ApJ, 456, L29

Mirabel, I. F. \& Rodríguez, L. F. 1999, ARA\&A, 37, 409

Del Monte, E. 2010, $A \& \mathcal{E} A$, in press [astro-ph/1004.0849]

Moskalenko I. V. \& Karakula S., 1994, ApJ, 92, 567

Orellana, M. et al. 2007, A\&A, 476, 9

Owocki, S., Cohen, P., \& David, H. 2006, ApJ, 648, 565

Paredes, J. M. et al. 2000, Science, 288, 2340

Paredes, J. M., Bosch-Ramon, V., \& Romero, G. E. 2006, A\&A, 451, 259

Perucho, M. \& Bosch-Ramon, V. 2008, A\&A, 482, 917

Perucho, M., Bosch-Ramon, V., \& Khangulyan, D. 2010a, A\&A, 512, L4

Perucho, M., et al. 2010b, $A \mathscr{E} A$, in press [astro-ph/1005.4332]

Protheroe, R. J. \& Stanev, T. 1987, ApJ, 322, 838

Ribó, M. 2005, in Future Directions in High Resolution Astronomy: The 10th Anniversary of the VLBA, (ASPC, 2005) 340, 421 [astro-ph/0402134]

Rieger, F. M., Bosch-Ramon, V., \& Duffy, P. 2007, Ap\&SSS, 309, 119

Romero, G. E. et al. 2003, A\&A, 410, L1

Romero, G. E. et al. 2007, A\&SA, 474, 15

Romero, G. E. \& Vila G. S. 2009, $A \& A$, 494, L33

Romero, G. E., Vieyro, F. L., \& Vila G. S. 2010, $A \& A$, in press [astro-ph/1006.5005]

Sabatini et al. 2010, ApJ, 712, L10

Saito et al. 2009, Proc. 31st ICRC

Sierpowska-Bartosik, A. \& Torres, D. F. 2007, ApJ, 671, L145

Takahashi, T. et al. 2009, ApJ, 697, 592

Tavani, M., et al. 2009a, Nature, 462, 620

Tavani, M., et al. 2009b, ApJ, 698, L142

Zdziarski, A. A., Malzac, J., \& Bednarek, W. 2009, MNRAS, 394, L41

\section{Discussion}

CAstro-Tirado: The compact sources detected at VHE are all high-mass X-ray binaries? Is this mainly due to the strong stellar winds from the companion stars?

Bosch-RAmon: The strong stellar wind should be relevant but the difference brought by the star, I think, is the stellar radiation field in the context of VHE production. 
KUnDT: You've been rightly stressing the impressive hardness of (some of) the microquasar spectra, with electron energies reaching PeV. An often mentioned "in-situ acceleration" conflict with the Second Law, beyond the test-particle limit, increasingly so for increasing Lorentz factors $\gamma \approx 10^{9}$. I encourage your consideration of an obliquely spinning magnetized neutron star as the exclusive booster, analogous to the Crab nebula.

BOSCH-RAMON: I agree with you that mechanisms of particle acceleration different from the canonical Fermi-type ones should be explored, since the conditions in these sources may indeed require something exotic to explain the very high energies of the observed photons. 